\title{
画HAD
}

ISSN-L: 2530-5115

DOI: http://doi.org/10.22585/hospdomic.v1i2.15

\section{Nutrición parenteral domiciliaria en los pacientes oncológicos}

\author{
Home Parenteral Nutrition in cancer patients
}

Carmina Wanden-Berghe', Cristina Cuerda Compes², Julia Álvarez Hernandez ${ }^{3}$ José Luis Pereira Cunill ${ }^{4}$, Fátima Carabaña Pérez ${ }^{5}$, Carmen Gómez Candela ${ }^{6}$; Grupo NADYA-SENPE.

1. Hospital General Universitario e ISABIAL-FISABIO, Alicante, España.

2. Hospital Gregorio Marañón, Madrid, España.

3. Hospital Universitario Príncipe de Asturias, Alcalá de Henares, Madrid, España.

4. Hospital Universitario Virgen del Rocío, Sevilla, España.

5. Hospital Ramón y Cajal, Madrid, España.

6. Hospital La Paz, Madrid, España.

Correspondencia/Correspondence

Carmina Wanden-Berghe

Hospital General Universitario de Alicante.

Instituto de Investigación Sanitaria y Biomédica

de Alicante (ISABIAL-Fundación FISABIO),

Alicante, España.

carminaw@telefonica.net
Recibido/Received

03.04 .2017

Aceptado/Accepted

03.04 .2017

CÓMO CITAR ESTE TRABAJO | HOW TO CITE THIS PAPER

Wanden-Berghe C, Cuerda Compes C, Álvarez Hernandez J, Pereira Cunill JL, Carabaña Pérez F, Gómez Candela C, et al; Grupo NADYA-SENPE. Nutrición parenteral domiciliaria en los pacientes oncológicos. Hosp Domic. 2017; 1(2):65-72. 


\section{RESUMEN}

Objetivo: analizar las características de los pacientes oncológicos con nutrición parenteral domiciliaria (NPD) recogidos en el registro NADYA.

Método: estudio transversal de los datos recogidos en el registro del grupo de nutrición artificial domiciliaria y ambulatoria NADYA de pacientes con NPD y diagnóstico oncológico explicito, desde el 1 de enero de 2010 al 31 de diciembre de 2016.

Resultados: en los 7 años estudiados se han recogido 1463 pacientes, de los que 599 han tenido un diagnóstico de cáncer $(40,94 \%)$ con un incremento del $43 \%$ desde el inicio. La edad media fue de 57 años IIQ [46 - 64] registrándose 5 niños. La mayoría de los pacientes eran oncológicos paliativos (51,3\%). Se registraron 3,18 complicaciones sépticas relacionadas con el catéter por cada 1000 días de nutrición, con mayor frecuencia en el grupo de enteritis rádica (ANOVA $p<0,001$ ). La principal causa de finalización fue el fallecimiento $61 \%$, los pacientes paliativos en mayor proporción que los demás diagnósticos $\left(x^{2}=53.819 ; p<0,001\right)$.

Conclusiones: Ios paliativos representan la mayoría de los pacientes oncológicos con NPD, siendo la obstrucción intestinal y el síndrome de intestino corto los motivos de indicación de casi el $80 \%$ de los casos. Los pacientes con enteritis rádica han sido los que menos complicaciones metabólicas y sépticas no relacionadas con el catéter presentaron, siendo los que más complicaciones sépticas relacionadas con el catéter. La calidad de vida sería un parámetro que proporcionaría una información muy valiosa en estos pacientes, siendo una limitación de este estudio no disponer de datos al respecto.

Palabras clave: Nutrición Parenteral Domiciliaria; Neoplasias; Nutrición Parenteral; Sistemas de Información.

\section{ABSTRACT}

Objective: to analyze the characteristics of oncology patients with home parenteral nutrition HPD collected from the NADYA registry.

Method: cross-sectional study of the data collected from the NADYA's group registry related to home and outpatient artificial nutrition. Patients with HPD and explicit oncological diagnosis were selected. Data were collected from January 1, 2010 to December 31, 2016.

Results: Within the 7 years studied, 1463 patients were collected, of which 599 had a cancer diagnosis (40.94\%) with an increase of $43 \%$ from the beginning. The mean age was 57 years IIQ [46-64], registering 5 children. The majority of the patients were oncologic palliative $(51.3 \%)$. There were 3.18 septic catheterrelated complications per 1000 days of nutrition, most frequently in the radiation enteritis group (ANOVA $p<0.001$ ). The main cause of end of treatment was death $61 \%$, palliative patients in higher proportion than the other diagnoses $\left(x^{2}=\right.$ 53,819, $\mathrm{p}<0.001)$.

Conclusions: palliative represent the majority of cancer patients with HPD. Intestinal obstruction and short bowel syndrome was the main reason for indicating it, almost $80 \%$ of cases. Patients with radiation enteritis were the ones with the least metabolic and septic complications not related to the catheter, being the ones with the most septic complications related to the catheter. Quality of life is a parameter that would provide a very valuable information of these patients. It is a limitation of this study not to have data based on this parameter.

Keywords: Home Parenteral Nutrition; Neoplasms; Parenteral Nutrition; Information Systems. 


\section{INTRODUCCIÓN}

La Nutrición Parenteral Domiciliaria (NPD) comenzó en unos pocos hospitales universitarios estadounidenses a finales de los años sesenta y principios de los setenta.La mayoría de estos pacientes tenían síndrome del intestino corto extremo condicionado por enfermedades benignas, tales como infarto vascular mesentérico o enfermedad de Crohn (1).

Aunque, la NPD surge como una técnica en el domicilio para procurar la supervivencia, con la máxima calidad de vida posible, de los pacientes con enfermedades «relativamente estables y de buen pronóstico vital» consiguiendo reinsertándolos en su ambiente familiar,e incluso social y laboral en algunas ocasiones (2). Hay otro grupo de pacientes que necesitan NPD de forma transitoria o permanente, cuyo diagnóstico basal es oncológico, y que puede tener un pronóstico de supervivencia bueno, o no tan bueno, pero en los que es posible mejorar su calidad de vida con este tratamiento. Además, todos los tipos de tratamiento contra el cáncerestán mejorando en efectividad y precisión. Por lo que, aunque muchos cánceres todavía no se pueden curar pueden convertirse en enfermedades crónicas (3).

El proceso oncológico en sí mismo, los síntomas que genera y también los que se asocian a los diferentes tratamientos, que se le aplican con fines curativos o paliativos, constituyen un elevado riesgo de desnutrición para los pacientes con cáncer.

La desnutrición va a incidir en el sujeto vulnerable, produciendo un gran deterioro de su estado funcional y un aumento de las complicaciones: infecciones, falta de respuesta al tratamiento, dehiscencia de suturas, disminución de la tolerancia al tratamiento quimioterápico, alteración de la imagen corporal, etcétera. Todo ello, va a determinar una disminución de la calidad de vida del paciente y una disminución del tiempo de supervivencia. La desnutrición progresiva hasta llegar a la caquexia ocurre frecuentemente en pacientes con cáncer, siendo éstas indicadores de mal pronóstico yresponsables de un incremento de su morbilidad y mortalidad. La caquexia se asocia a una menor mediana de supervivencia, a una peor respuesta a la quimioterapia y a un mal estado general del paciente (4).

El enorme crecimiento que ha tenido la nutrición artificial domiciliaria (NAD) en las últimas décadas, tanto enteral como parenteral, ha hecho necesario el desarrollo de sistemas de información que recoja los datos de los pacientes que se benefician de este tratamiento. Gracias a estos registros podemos aproximarnos a conocer la prevalencia de la NAD en distintos países (5), las indicaciones más frecuentes, las complicaciones principales, la supervivencia, etcétera. Aplicando esta información a los diferentes ámbitos se pretende conseguir una mejora de la calidad asistencial (6-8).

El Grupo de Nutrición Artificial Domiciliaria y Ambulatoria (NADYA) perteneciente a la Sociedad Española de Nutrición Parenteral y Enteral (SENPE) mantiene desde 1992 un registro para pacientes con nutrición artificial domiciliaria (enteral y parenteral), publicándose periódicamente los informes que abarcan los datos incluidos desde su inicio hasta la actualidad. El propósito de este trabajo es presentar el análisis de la información sobre los pacientes oncológicos con NPD recogidos en el registro NADYA (9).

\section{MÉTODOS}

Estudio descriptivo transversal de los datos recogidos en el registro del grupo NADYA-SENPE (www.nadya-senpe.com). Se incluyeron en el estudio los pacientes con NPD y diagnóstico oncológico explicito, desde el 1 de enero de 2010 al 31 de diciembre de 2016. 
Para el análisis de los datos se consideró como población pediátrica «niños» desde la edad más pequeña registrada hasta los 14 años, considerándose adultos las edades superiores. Las variables cualitativas se describieron con su frecuencia y porcentaje, las cuantitativas con medidas de tendencia central: media (desviación estándar) o mediana (intervalo interquartílico IIQ) según presentaran, o no, una distribución normal. Para comprobar la distribución de las variables y su ajuste a la normalidad se utilizara la prueba de Kolmogorov-Smirnov. Para los sucesivos análisis del efecto de las variables recogidas se utilizarán los test Chi cuadrado, T-Student, ANOVA, U de Mann-Whitney y Kruskal-Wallis dependiendo de las características de las variables. Para el cálculo de las prevalencias se tomó como denominador la media poblacional a fecha 1 de julio de cada uno de los años estudiados, publicada por el Instituto Nacional de Estadística (http://www.ine.es). El control de calidad de los datos se efectuó a través de tablas de doble entrada y búsqueda activa de errores, cuando fueron encontrados se corrigieron mediante la consulta con la fuente original. Para el análisis se empleó el paquete estadístico Statistical Package for the Social Sciences (SPSS ${ }^{\circledR}$ versión 22.0).

\section{RESULTADOS}

Los datos obtenidos muestran que la NPD en el paciente oncológico en España, ha tenido una evolución discretamente ascendente a lo largo de los años, con un incremento en 7 años del 43\%, al igual que el resto de los diagnósticos que motivan la indicación que han aumentado el 36\% (figura 1). Durante estos 7 años se recogieron un total de 1463 pacientes, de los que 599 tuvieron un diagnóstico de cáncer (40,94\%), siendo el 58,30\% mujeres.

La edad no siguió una distribución normal (Kolmogorov-Smirnov $=0,07 ; p<0,001$ ), la media (Me) fue de 57 años IIQ [46 - 64] con un mínimo de 3 años y máximo de 88, contabilizándose 5 niños y siendo la distribución de la edad la misma en ambas categorías de sexo ( $U$ de Mann-Whitney = 39842,50; $p=0,07$ ).

Durante estos años se registraron 623 episodios de NPD ya que 12 pacientes presentaron 2, y 2 pacientes 3 episodios. La duración Me de los episodios fue de 123 días IIQ [41 - 316] siendo la moda de 77 días y con un máximo de duración de 3423 días (9,40 años). La procedencia geográfica de los enfermos de los 36 hospitales participantes se muestra en la figura 2.

El diagnóstico de inicio de la NPD en los pacientes oncológicos, se distribuyó en tres grupos: paliativos (51,3\%), en tratamiento activo y enteritis radica (figura 3). El motivo de la indicación fue en el $46,8 \%$ de los pacientes por obstrucción intestinal, seguido por el síndrome de intestino corto 30,3\% (figura 4). La vía de acceso de la NPD fue el catéter tunelizado en el 41,4\% de los casos, se utilizó un catéter implantado en el 37,5\% y el catéter central de inserción periférica (PICC) en el 21,1\% de los casos. Los pacientes con catéter tunelizado presentaban una edad significativamente mayor a los que tenían catéter implantado $(p=0,001)$, y los que tenían PICC no presentaron diferencias con los otros dos grupos. Siendo los pacientes paliativos los que en mayor proporción utilizan el catéter implantado y los que tienen enteritis radica con mayor frecuencia el catéter tunelizado $\left(x^{2}=94.208\right.$; $p<0,001)$. Dos de los pacientes también eran portadores de yeyunostomía $(2,80 \%)$.

Los pacientes presentaron 143 complicaciones metabólicas (1,33/1000 días de nutrición), complicaciones sépticas no relacionadas con el catéter 113 (1,05/1000 días de nutrición) siendo el grupo de enteritis rádica el que menos complicaciones de ambos tipos presentó $(p<0,001)$ y se registraron 342 complicaciones sépticas relacionadas con el catéter (3,18/1000 días de nutrición) siendo el grupo de enteritis rádica el que más presentó $(p<0,001)$. 
El grado de funcionalidad en lo referente a la actividad y la autonomía de los pacientes eran normalesen el 22,50\% y 35,10\% respectivamente al inicio del tratamiento nutricional (figura 5).

Finalizaron $323(53,90 \%)$ de los episodios de NPD siendo la principal causa el fallecimiento $61,00 \%$ y el $25,40 \%$ recuperó la vía oral, los pacientes paliativos fallecieron significativamente en mayor proporción que los demás diagnósticos $\left(x^{2}=53.819 ; p<0,001\right)$.

\section{DISCUSIÓN}

Este trabajo presenta la serie más amplia de pacientes con NPD y diagnóstico de cáncer en nuestro país. Al igual que en otros registros se ha observado un incremento en el número de pacientes con este tratamiento en su domicilio aunque quizá no tan elevado como en otros países, como Estados Unidos en que observaron un incremento del 25\% por año, registrando 10.101 paciente en un periodo de 6 años (1985-90). Estiman que el 38,70\% tienen diagnóstico oncológico, aunque en ellos solo distinguen entre cáncer activo y enteritis por radiación (1). A pesar de que el uso de la NPD continúa siendo controvertido y que está asociado a una importante carga emocional, cultural y filosófica, el cáncer continúa siendo la principal indicación de NPD tanto en series europeas (10) como norteamericanas (11).

El registro NADYA no recoge la evaluación de la calidad de vida (CV) de los pacientes, lo que se considera una limitación, fundamentalmente en el estudio de la NPD en este tipo de pacientes. En cuanto al nivel de autonomía y de actividad, como dimensiones que integran la CV, se pudo apreciar que la mayoría de los pacientes que reciben NPD, a pesar de ser un tratamiento con una técnica compleja, mantuvieron un alto nivel de autonomía. Algunos estudios que evalúan la CV llegan a la conclusión de que hay un deterioro de la CV asociada a este tratamiento (12-15), aunque la evolución de la enfermedad y el impacto del tratamiento pueden tener un efecto confusor importante para llegar a esta conclusión. En un reciente estudio Delphi, para establecer los criterios excelencia en la calidad asistencial en estos pacientes con NPD, en el que participaron 27 expertos de 5 países europeos, se llegó a la conclusión de que los 3 indicadores principales fueron: la calidad de vida, la incidencia de reingreso hospitalario y la incidencia de las infecciones relacionadas con el catéter (16).

En este estudio, la incidencia de complicaciones sépticas relacionadas con el catéter fue mayor que en otras series del registro NADYA(17) y los pacientes con enteritis rádica fueron los que menos complicaciones metabólicas y sépticas no relacionadas con el catéter presentaron. Sin embargo, fueron los que tuvieron más complicaciones sépticas relacionadas con el catéter. En Canadá se documentaron 1,58 sepsis/1.000 días relacionadas con el catéter de 2005 a 2008 y 0,97/1.000 días de NPD entre 2011 y $2014(17,18)$; en otra serie europea Guglielmi y cols. (20) registraron 1,40 sepsis por catéter/1.000 días de NPD con mayor incidencia en pacientes oncológicos. Se podría plantear si realmente este colectivo de pacientes tiene mayor número de complicaciones o si lo que se viene cuestionando desde hace años como un sesgo de recogida de esta variable en el registro NADYA podría ser reconsiderado.

En consecuencia, se podría concluir: en los 7 años estudiados el número de pacientes oncológicos con NPD ha ido aumentando. Los oncológicos paliativos representan la mayoría de los pacientes, siendo la obstrucción intestinal y el síndrome de intestino corto los motivos de indicación de casi el $80 \%$ de los casos. Los pacientes con enteritis rádica han sido los que tuvieron más complicaciones sépticas relacionadas con el catéter, sin embargo los que menos complicaciones metabólicas y sépticas no relacionadas con el catéter presentaron. 
La CV es un parámetro que proporcionaría una información muy valiosa en estos pacientes, siendo una limitación de este estudio no disponer de datos al respecto.

\section{AGRADECIMIENTOS}

A los profesionales que colaboran registrando sus pacientes con NPD en el grupo NADYA: Rosa Burgos Peláez, Luisa Chicharro y Victoria Avilés del Hospital de la Vall d'Hebrón, Barcelona. Laura Frías Soriano y Nerea Fernández Colmenar del Hospital Gregorio Marañón, Madrid. Cristina Campos Martín del Hospital Virgen Macarena, Sevilla. Antonio Pérez de la Cruz y Gabriela Lobo Támer del Hospital Virgen de las Nieves, Granada. Nuria Virgili Casas, Rafael López Urdiales y Enric Roca Rocadel Hospital Bellvitge, l'Hospitalet de Llobregat, Barcelona. Ceferino Martinez Faedo del Hospital Central de Asturias, Oviedo. María José Martínez Ramírez y Carmen Arraiza Irigoyen del Complejo Hospitalario de Jaén. Carmen Garde Orbaiz del Hospital Donostia, Guipúzcoa. Ma Angeles Penacho Lázaro del Hospital El Bierzo, Ponferrada, León. José Manuel Moreno Villares y Carmelo Loinaz Segurola del Hospital 12 de Octubre, Madrid. Eva Angeles Sánchez Martos de la Corporació Sanitària Parc Taulí, Barcelona. José Antonio Irles Rocamora y Yolanda Borrego Izquierdo del Hospital Nuestra Señora de Valme, Sevilla. Alejandro Sanz Paris del Hospital Miguel Servet, Zaragoza. Montserrat Gonzalo Marín del Hospital Carlos Haya, Málaga. Ana Zugasti Murillo del Hospital Virgen del Camino, Pamplona. Pedro Pablo García Luna, Juana Parejo y Diana Ariadel Cobo del Hospital Virgen del Rocío, Sevilla. Pilar Matía Martín y Miguel Angel Rubio Herrera del Hospital Clínico San Carlos, Madrid. Tomás Martín Folgueras del Complejo Hospitalario de Canarias, Santa Cruz de Tenerife. Esther Ramos Boluda, Samara Palma Milla, Laura Zurita Rosa y Diego Rodriguez Duran del Hospital de la Paz, Madrid. Patricia Díaz Guardiola del Hospital Infanta Sofía, San Sebastián de los Reyes, Madrid. Cristina Tejera Pérez del Complejo Hospitalario de Ferrol, A Coruña. Daniel de Luis Román del Hospital Clínico, Valladolid. Luis Miguel Luengo Pérez del Hospital Infanta Cristina, Badajoz. Nieves Santacruz Carmona del Hospital General, Alicante. M ${ }^{a}$ Ángela Martin Palmero del Hospital San Pedro, Logroño. Antxón Apezetxea Celaya del Hospital Basurto, Bilbao. Miguel Angel Ponce Gonzalez del Hospital de Gran Canaria Dr. Negrín, Las Palmas de Gran Canaria. Maria de los Ángeles Martin Fontalba del Hospital Virgen de la Victoria, Málaga. Rebeca Sanchez Sanchez del Hospital de Cruces, Bilbao. Miguel Angel Martinez Olmos del Complejo Hospitalario, Santiago de Compostela. Olga Sánchez-Vilar Burdiel, de la Fundación Jiménez Díaz, Madrid. Clara Joaquín Ortiz del Hospital Germans Trias i Pujol, Barcelona. Cecilia Martínez Costa del Hospital Clínico, Valencia. José Pablo Suárez Llanos, Francisca Pereyra-García Castro y Carmen Fraile Clemente del Hospital Nuestra Señora de la Candelaria, Santa Cruz de Tenerife. Alicia Calleja Fernández y Alfonso Vidal Casariego del Complejo Asistencial, León. Pere Leyes García, María de Talló Forga Visa y Joan Trabal Vílchez del Hospital Clinic, Barcelona. Mª Carmen Gil Martinez del Hospital Central de la Defensa Gomez Ulla, Madrid. Silvia Mauri del Hospital Dr. Josep Trueta, Girona. Maria Victoria García Zafra del Hospital Virgen de la Arrixaca, Murcia. Rosa Mª Parés Marimón del Consorci Sanitari de l'Anoia, Igualada, Barcelona. Maria José Carrera Santaliestra del Hospital del Mar, Barcelona. 


\section{BIBLIOGRAFÍA}

1. Howavd L. Home Parenteral and Enteral Nutrition in Cancer Patients. Cancer. 1993;72(11 Suppl):3531-41. PMID: 8242585

2. Álvarez Hernández J, Muñoz Carmona D, Planas Vila M, Rodríguez Rodríguez I, Sánchez P. Criterios de intervención nutricional: objetivos, indicaciones y evidencias. Nutr Hosp Suplementos. 2008;1(1):26-33.

3. Arends J, Bachmann P, Baracos V, Barthelemy N, Bertz H, Bozzetti F, et al. ESPEN guidelines on nutrition in cancer patients. Clin Nutr. 2017;36(1):11-48. DOI: 10.1016/j.clnu.2016.07.015; PMID: 27637832

4. Bozzetti F, Braga M, Gianotti L, Gavazzi C, Mariani L. Postoperative enteral versus parenteral nutrition in malnourished patients with gastrointestinal cancer: a randomized multicenter trial. Lancet. 2001;358(9292):1487-92. DOI: 10.1016/S0140-6736(01)06578-3; PMID: 11705560

5. Castelló-Botía I, Wanden-Berghe C, Sanz-Valero J. Artificial nutritional support registries: systematic review. Nutr Hosp. 2009;24(6):711-6. DOI:10.3305/nh.2009.24.6.4556; PMID: 20049375

6. Wanden-Berghe C, Sanz-Valero J, Culebras J; Red Mel-CYTED. Información en Nutrición Domiciliaria: la importancia de los registros. Nutr Hosp. 2008;23(3):220-5. PMID: 185606985

7. Luengo Pérez LM, Guerra Martínez S, Georgescu L.Nutrición enteral domiciliaria prescrita en el Hospital Universitario Infanta Cristina de Badajoz (España). Hosp Domic. 2017;1(1):35-45. DOI:10.22585/hospdomic.v1i1.4

8. Wanden-Berghe C, Pérez de la Cruz A, Lobo G, Calleja A, Gómez C, Zugasti A, et al; Grupo NADYA-SENPE. El registro de Nutrición Artificial Domiciliaria y Ambulatoria de la Sociedad Española de Nutrición Parenteral y Enteral: análisis DAFO. Nutr Hosp. 2012;27(4):1357-60. DOI: 10.3305/nh.2012.27.4.5912; PMID: 23165586

9. Wanden-Berghe C, Pereira Cunill JL, Cuerda Compes C, Moreno Villares JM, Pérez de la Cruz A, Burgos Peláez R, et al; NADYA-SENPE. Nutrición parenteral domiciliaria en España durante 2014; informe del Grupo de Nutrición Artificial Domiciliaria y Ambulatoria NADYA. Nutr Hosp. 2015;32(6):2380-84. DOI: 10.3305/nh.2015.32.6.10056; PMID: 26667683

10. Bakker H, Bozzetti F, Staun M, Leon-Sanz M, Hebuterne X, Pertkiewicz M, et al. Home parenteral nutrition in adults: a European multicentre survey in 1997. Clin Nutr. 1999;18(3):135-40. DOI: 10.1054/cInu. 1999.0021; PMID: 10451476

11. Howard L, Ament M, Fleming CR, Shike M, Steiger E: Current use and clinical outcome of home parenteral and enteral nutrition therapies in the United States. Gastroenterology. 1995;109(2):355-65. PMID: 7615183

12. Oz V, Theilla M, Singer $P$. Eating habits and quality of life of patients receiving home parenteral nutrition in Israel. Clin Nutr. 2008;27(1):95-9. DOI: 10.1016/j.clnu.2007.06.016; PMID: 18045751

13. Pironi L, Paganelli F, Mosconi P, Morselli-Labate AM, Spinucci G, Merli C, et al. The SF-36 Instrument for the follow up of health-related quality-of-life assessment of patients undergoing home parenteral nutrition for benign disease. Transplant Proc. 2004;36(2):255-8. DOI: 10.1016/j. transproceed.2003.12.008; PMID: 15050126

14. Persoon A, Huisman-de Waal G, Naber TA, Schoonhoven L, Tas T, Sauerwein H, et al. Impact of long-term HPN on daily life in adults. Clin Nutr. 2005;24(2):304-13.

15. Carlsson E, Bosaeus I, Nordgren S. Quality of life and concerns in with short bowel syndrome. Clin Nutr. 2003;22(5):445-52. DOI: 10.1016/S0261-5614(03)00042-6; PMID: 14512031 
16. Dreesen M, Foulon V, Hiele M, Vanhaecht K, De Pourcq L, Pironi L, et al. Quality of care for cancer patients on home parenteral nutrition: development of key interventions and outcome indicators using a two-round Delphi approach. Support Care Cancer. 2013;21(5):1373-81. DOI: 10.1007/s00520-012-1679-1; PMID: 23229653

17. Wanden-Berghe C, Campos Martín C, Cuerda Compes C, Gómez Candela C, Burgos Peláez R, Moreno Villares JM; Grupo NADYA-SENPE. Nutrición parenteral domiciliaria en España durante 2015; informe del Grupo de Nutrición Artificial Domiciliaria y Ambulatoria NADYA. Nutr Hosp. 2016;33(6):1487-90. DOI: http://dx.doi.org/10.20960/nh.464

18. Hortencio TD, Arendt BM, Teterina A, Jeejeebhoy KN, Gramlich LM, WhittakerJS, et al. Changes in home parenteral nutrition practice based on the Canadian Home Parenteral Nutrition Patient Registry. JPEN J Parenter Enteral Nutr. 2015; sep 25. Epub ahead of print. DOI: 10.1177/0148607115609289; PMID: 26407599

19. Winkler MF, DiMaria-Ghalili RA, Guenter P, Resnick HE, Robinson L, LymanB, et al. Characteristics of a cohort of home parenteral nutrition patients at the time of enrollment in the Sustain Registry. JPEN J Parenter Enteral Nutr. 2016 40(8):1140-49. DOI: 10.1177/0148607115586575; PMID: 25972431

20. Guglielmi FW, Regano N, Mazzuoli S, Rizzi M, Fregnan S, Leogrande G, et al. Catheter-related complications in long-term home parenteral nutrition patients with chronic intestinal failure. J Vasc Access. 2012;13(4):490-7. DOI: 10.5301/jva.5000133; PMID: 23258588 